\title{
Definition of competence standards for optical diagnosis of diminutive colorectal polyps: European Society of Gastrointestinal Endoscopy (ESGE) Position Statement
}

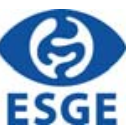

Authors

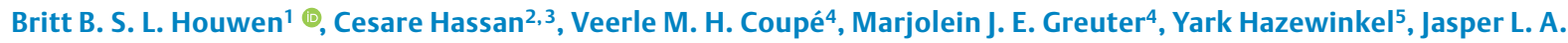
Vleugels ${ }^{1}$, Giulio Antonelli6,7, Marco Bustamante-Balén ${ }^{8,9} \odot$, Emmanuel Coron ${ }^{10}$, George A. Cortas ${ }^{11}$, Mario DinisRibeiro ${ }^{12,13}$, Daniela E. Dobru ${ }^{14}$, James E. East ${ }^{15,16}$, Marietta lacucci ${ }^{17}$, Rodrigo Jover ${ }^{18}$, Roman Kuvaev ${ }^{19,20}$, Helmut Neumann 21,22 ๑, Maria Pellisé ${ }^{23,24} \odot$, Ignasi Puig ${ }^{25,26} \odot$, Matthew D. Rutter ${ }^{27,28} \odot$, Brian Saunders $^{29}$, David J. Tate $^{30,31}$, Yuichi Mori ${ }^{32,33,34} \oplus$, Gaius Longcroft-Wheaton ${ }^{35}$, Raf Bisschops ${ }^{36} \bullet$, Evelien Dekker ${ }^{1} \oplus$

Institutions

1 Department of Gastroenterology and Hepatology, Amsterdam University Medical Center, location AMC, University of Amsterdam, Amsterdam, The Netherlands

2 Department of Biomedical Sciences, Humanitas University, Pieve Emanuele, Milan, Italy

3 Endoscopy Unit, IRCCS Humanitas Clinical and Research Center, Rozzano, Milan, Italy

4 Department of Epidemiology and Data Science, Amsterdam University Medical Center, location VUmc, Amsterdam, The Netherlands

5 Department of Gastroenterology and Hepatology, Radboud University Medical Center, Radboud University, Nijmegen, The Netherlands

6 Department of Anatomical, Histological, Forensic Medicine and Orthopedics Sciences, "Sapienza” University of Rome, Rome, Italy

7 Gastroenterology and Digestive Endoscopy Unit, Ospedale dei Castelli Hospital, Ariccia, Rome, Italy

8 Gastrointestinal Endoscopy Unit, Digestive Diseases Department, La Fe Polytechnic University Hospital, Valencia, Spain

9 Gastrointestinal Endoscopy Research Group, La Fe Health Research Institute, Valencia, Spain

10 Institut des Maladies de l'Appareil Digestif, Nantes, France

11 Division of Gastroenterology, University of Balamand, Faculty of Medicine, St. George Hospital University Medical Center, Beirut, Lebanon

12 Porto Comprehensive Cancer Center (Porto.CCC), Porto, Portugal

13 RISE@CI-IPOP (Health Research Network), Porto, Portugal
14 Gastroenterology Department, George Emil Palade University of Medicine, Pharmacy, Science and Technology of Targu Mures, Targu Mures, Romania

15 Translational Gastroenterology Unit, Nuffield Department of Medicine, Experimental Medicine Division, John Radcliffe Hospital, University of Oxford, Oxford, UK

16 Division of Gastroenterology and Hepatology, Mayo Clinic Healthcare, London

17 Institute of Translational of Medicine, Institute of Immunology and Immunotherapy and NIHR Biomedical Research Centre, University of Birmingham and University Hospitals Birmingham NHS Foundation Trust, Birmingham, UK

18 Servicio de Medicina Digestiva, Hospital General Universitario de Alicante, Instituto de Investigación Sanitaria ISABIAL, Universidad Miguel Hernández, Alicante, Spain

19 Endoscopy Department, Yaroslavl Regional Cancer Hospital, Yaroslavl, Russian Federation

20 Department of Gastroenterology, Faculty of Additional Professional Education, N.A. Pirogov Russian National Research Medical University, Moscow, Russian Federation

21 Department of Medicine I, University Medical Center Mainz, Mainz, Germany

22 GastroZentrum, Lippe, Germany

23 Department of Gastroenterology, Hospital Clínic de Barcelona, Barcelona, Spain

24 Centro de Investigación Biomédica en Red de Enfermedades Hepáticas y Digestivas (CIBERehd), Institut d'Investigacions Biomèdiques August Pi i Sunyer (IDIBAPS), Universitat de Barcelona, Barcelona, Spain

25 Digestive Diseases Department, Althaia Xarxa Assistencial Universitària de Manresa, Manresa, Spain 
Department of Medicine, Facultat de Ciències de la Salut, Universitat de Vic-Universitat Central de Catalunya (UVic-UCC), Manresa, Spain

27 Faculty of Medical Sciences, Newcastle University, Newcastle-upon-Tyne, UK

28 University Hospital of North Tees, Stockton-on-Tees, UK

29 Department of Gastroenterology, St Mark's Hospital and Academic Institute, Harrow, UK

30 Department of Gastroenterology and Hepatology, University of Ghent, Ghent, Belgium

31 University Hospital Ghent, Ghent, Belgium

32 Clinical Effectiveness Research Group, Institute of Health and Society, University of Oslo, Oslo, Norway

33 Section of Gastroenterology, Department of Transplantation Medicine, Oslo University Hospital, Oslo, Norway

34 Digestive Disease Center, Showa University Northern Yokohama Hospital, Yokohama, Japan

35 Portsmouth Hospitals University NHS Trust, Cosham, Portsmouth, UK

36 Department of Gastroenterology and Hepatology, Catholic University of Leuven, (KUL), TARGID, University Hospital Leuven, Leuven, Belgium

published online 6.12 .2021

\section{Bibliography}

Endoscopy 2022; 54: 88-99

DOI 10.1055/a-1689-5130

ISSN 0013-726X

(C) 2021. European Society of Gastrointestinal Endoscopy

All rights reserved.

This article is published by Thieme.

Georg Thieme Verlag KG, Rüdigerstraße 14,

70469 Stuttgart, Germany

Tables $1 \mathrm{~s}-3 \mathrm{~s}$

Supplementary material is available under

https://doi.org/10.1055/a-1689-5130

Corresponding author

Evelien Dekker, MD PhD, Amsterdam University Medical

Center, location Academic Medical Center, Department of

Gastroenterology and Hepatology, Meibergdreef 9, 1105 AZ

Amsterdam, The Netherlands

e.dekker@amsterdamumc.nl

\section{ABSTRACT}

Background The European Society of Gastrointestinal Endoscopy (ESGE) has developed a core curriculum for high quality optical diagnosis training for practice across Europe. The development of easy-to-measure competence standards for optical diagnosis can optimize clinical decision-making in endoscopy. This manuscript represents an official Position Statement of the ESGE aiming to define simple, safe, and easy-to-measure competence standards for endoscopists and artificial intelligence systems performing optical diagnosis of diminutive colorectal polyps $(1-5 \mathrm{~mm})$.

Methods A panel of European experts in optical diagnosis participated in a modified Delphi process to reach consensus on Simple Optical Diagnosis Accuracy (SODA) competence standards for implementation of the optical diagnosis strategy for diminutive colorectal polyps. In order to assess the clinical benefits and harms of implementing optical diagnosis with different competence standards, a systematic literature search was performed. This was complemented with the results from a recently performed simulation study that provides guidance for setting alternative competence standards for optical diagnosis. Proposed competence standards were based on literature search and simulation study results. Competence standards were accepted if at least $80 \%$ agreement was reached after a maximum of three voting rounds.

Recommendation 1 In order to implement the leave-insitu strategy for diminutive colorectal lesions $(1-5 \mathrm{~mm})$, it is clinically acceptable if, during real-time colonoscopy, at least $90 \%$ sensitivity and $80 \%$ specificity is achieved for high confidence endoscopic characterization of colorectal neoplasia of $1-5 \mathrm{~mm}$ in the rectosigmoid. Histopathology is used as the gold standard.

Level of agreement $95 \%$.

Recommendation 2 In order to implement the resectand-discard strategy for diminutive colorectal lesions (1-5 $\mathrm{mm})$, it is clinically acceptable if, during real-time colonoscopy, at least $80 \%$ sensitivity and $80 \%$ specificity is achieved for high confidence endoscopic characterization of colorectal neoplasia of $1-5 \mathrm{~mm}$. Histopathology is used as the gold standard.

Level of agreement $100 \%$.

Conclusion The developed SODA competence standards define diagnostic performance thresholds in relation to clinical consequences, for training and for use when auditing the optical diagnosis of diminutive colorectal polyps. 


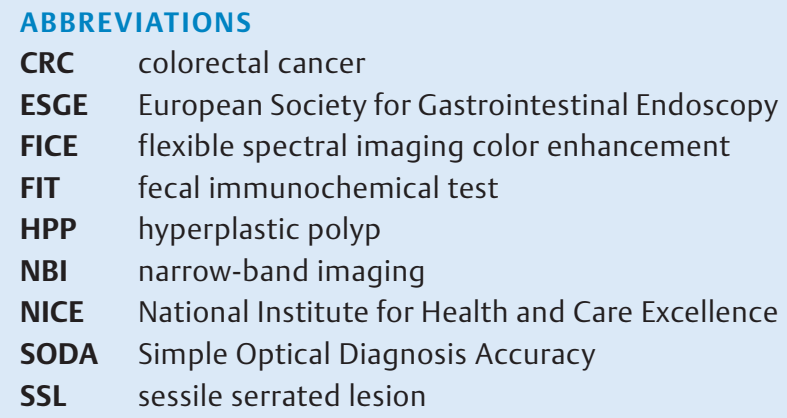

\section{SOURCE AND SCOPE}

This Position Statement is an official statement of the European Society of Gastrointestinal Endoscopy (ESGE). This position statement is the first in a series that will give details of new optical diagnosis standards. The recommendations presented here are based on a consensus procedure among endoscopists considered to be experts in optical diagnosis who are involved in optical diagnosis training and training courses in Europe.

\section{Introduction}

The European Society of Gastrointestinal Endoscopy (ESGE) has a vision to create a thriving community of endoscopy services and endoscopists in Europe, to provide a high quality of endoscopy care. ESGE has developed a core curriculum for optical diagnosis practice across Europe for high quality optical diagnosis training [1,2]. The development of easy-to-measure competence standards for optical diagnosis that are focused on clinical consequences could help to optimize clinical management in gastrointestinal endoscopy.

Recently, a spin-off task force of the Curricula Working Group for optical diagnosis training has developed a methodological framework, which can provide guidance when developing new competence standards for optical diagnosis in gastrointestinal endoscopy. The development of such standards could be a first step in optimizing clinical treatment and surveillance decisions within gastrointestinal endoscopy. As a first step, in 2021 , this task force started the initiative to develop competence standards for the optical diagnosis of diminutive colorectal polyps ( $1-5 \mathrm{~mm})$.

\section{Rationale for developing competence standards for optical diagnosis of diminutive colorectal polyps}

Diminutive colorectal polyps, with a negligible risk of harboring cancer, constitute up to $60 \%$ of all colorectal polyps $[3,4]$. The current management of polyps, including diminutive polyps, is to resect and submit them all for histological assessment. While the detection and removal of polyps contributes toward the reduction in colorectal cancer (CRC), histological assessment of these diminutive polyps results in substantial burdens and costs for colonoscopy units. These costs and burdens could be reduced by implementing an "optical diagnosis strategy" for diminutive colorectal polyps [5]. In this optical diagnosis strategy, endoscopists or artificial intelligence systems diagnose diminutive polyps during colonoscopy with high or low confidence. When these diminutive polyps are diagnosed with high confidence, they can be resected and discarded without histological evaluation (i.e. the "resect-and-discard strategy"). In addition, non-neoplastic lesions located in the rectum and sigmoid can be left in situ without resection, as they have no malignant potential (i.e. the "leave-in-situ strategy") [6, 7].

Though not yet achieved, the implementation of optical diagnosis for diminutive colorectal polyps into routine clinical practice remains an important goal as it would greatly reduce colonoscopy-associated costs $[8,9]$. The implementation of this strategy is therefore also endorsed by several international societies [10-12].

However, because misdiagnosis of diminutive lesions can result in inappropriate surveillance intervals and neoplastic lesions being left in situ, proficiency in optical diagnosis must be guaranteed before implementation. To address this, the ESGE Curricula Working Group for optical diagnosis training has comprehensively described the major training steps to achieve and maintain proficiency in optical diagnosis of diminutive colorectal polyps [1,2]. ESGE suggests that an endoscopist is competent after attending a validated training course, including an in vivo phase, and after reaching the endorsed competence standards during real-time colonoscopy. The currently endorsed PIVI competence criteria are however impractical and difficult to implement in daily practice. Developing alternative, easy-tomeasure competence criteria might facilitate the implementation of the optical diagnosis strategy in clinical practice.

\section{Aims}

With this modified Delphi procedure, the European Society of Gastrointestinal Endoscopy (ESGE) aimed to reach consensus on evidence-based competence standards for the optical diagnosis of diminutive colorectal polyps that are clinical acceptable, achievable, relevant, and easy to measure in daily practice. These standards are defined as the Simple Optical Diagnosis Accuracy (SODA) standards.

\section{Methodology}

A panel of European experts in optical diagnosis was asked to participate in a modified Delphi process to reach consensus on competence standards for the optical diagnosis of diminutive colorectal lesions (the SODA competence standards) [13-15]. This panel consisted of experts from the 2019 ESGE guideline group on advanced imaging for the detection and differentiation of colorectal neoplasia [11] and the ESGE Curriculum Working Group on optical diagnosis training [2]. During an online meeting, experts were introduced to the methodology of the Delphi procedure [13-15]. In addition, the methodology and results of a recent simulation study that provides guidance 
for setting alternative competence standards for the optical diagnosis of diminutive polyps were shown [16].

During the Delphi procedure, several competence standards for the optical diagnosis of diminutive polyps were proposed to the panel. For each proposed competence standard, the potential clinical benefits and harms of implementing the optical diagnosis strategy with this standard were shown. These potential benefits and harms were based on a systematic literature review, complemented by the results of a recent simulation study [16]. Because the clinical consequences of implementing the resect-and-discard strategy and the leave-in-situ strategy for diminutive colorectal polyps are different, the results and potential competence standards were presented separately. An extensive description of the methodology and Delphi procedure can be found in the methodological framework paper [17].

\section{SODA competence standards for implementation of the optical diagnosis strategy for diminutive colorectal polyps Leave-in-situ strategy}

\section{RECOMMENDATION}

1 In order to implement the leave-in-situ strategy for diminutive colorectal lesions $(1-5 \mathrm{~mm})$, it is clinically acceptable if, during real-time colonoscopy, at least $90 \%$ sensitivity and $80 \%$ specificity is achieved for high confidence endoscopic characterization of colorectal neoplasia of $1-5 \mathrm{~mm}$ in the rectosigmoid. Histopathology is used as the gold standard.

Level of agreement 95\% (14 Strongly agree; 7 Agree; 0 Neither agree nor disagree; 1 Disagree; 0 Strongly disagree).

The panel took into account a number of considerations in the development of this SODA competence standard for implementation of the leave-in-situ strategy, which are detailed in the following sections.

\section{Potential impact on short-term consequences}

To establish safe and easy-to-measure competence standards for the leave-in-situ strategy for diminutive colorectal lesions, decisions should be made on clinically acceptable short-term consequences of implementing this strategy. When applying the leave-in-situ part of the optical diagnosis strategy, not recognizing neoplastic diminutive lesions could result in neoplastic lesions being left in the rectosigmoid, whereas not recognizing non-neoplastic diminutive lesions could result in unnecessary polypectomies of non-neoplastic lesions in the rectosigmoid.

In addition, it could be argued that the correct diagnosis of each polyp subtype is not of equal clinical importance. The clinical implications of incorrectly diagnosing neoplastic lesions in the rectosigmoid (i. e. leaving in situ a neoplastic lesion) and incorrectly diagnosing non-neoplastic lesions in the recto- sigmoid (i.e. performing unnecessary polypectomy of a nonneoplastic lesion) are completely different. Therefore, from a patient safety point of view, correctly diagnosing non-neoplastic lesions may not be as clinically important as correctly diagnosing neoplastic lesions. However, from a time and cost-effectiveness point of view, correctly diagnosing non-neoplastic lesions is also important. Therefore, competence standards should take both scenarios into account.

To provide guidance for setting alternative competence standards for the optical diagnosis of diminutive colorectal polyps, a recent simulation study determined the relationship between the proportion of correctly optically diagnosed diminutive polyps and the above-mentioned short-term clinical consequences (i. e. [non]neoplastic lesions that would remain in situ in the rectosigmoid and surveillance interval agreement).

\section{SHORT DESCRIPTION OF THE SIMULATION APPROACH USED [16]. \\ In this simulation approach, a virtual endoscopist/an arti- ficial intelligence system performed optical diagnosis of diminutive polyps with a fixed diagnostic performance level ("strategy") on two existing cohorts of patients who underwent colonoscopy in either a primary colonos- copy screening or fecal immunochemical test setting $[18,19]$. A total of 756 strategies were defined by system- atically varying the proportion of correct optical diagno- ses for each polyp subtype (i.e. adenomas, sessile serra- ted lesions, hyperplastic polyps). For each strategy the short-term clinical consequences (i. e. surveillance inter- val agreements, number of [non]neoplastic lesions left in situ in the rectosigmoid) were determined using Monte Carlo Sampling with 1000 repetitions.}

This simulation study showed that the proportion of correctly diagnosed diminutive polyps (i. e. adenomas, sessile serrated lesions [SSLs], hyperplastic polyps [HPPs]) could be used as easy-to-implement competence standards for the performance of optical diagnosis of diminutive polyps. However, the question remained as to what an endoscopy society would consider clinically acceptable competence standards. Different proportions of correctly diagnosed diminutive polyps lead to different clinical consequences depending on the clinical setting and the surveillance guidelines used.

- Table 1 shows the short-term clinical consequences of implementing the leave-in-situ strategy using a range of settings for the proportion of correctly optically diagnosed adenomas and HPPs in a primary colonoscopy and fecal immunochemical test (FIT)-positive screening cohort. Results in this table are presented per 1000 individuals with at least one diminutive polyp. The impact on the number of diminutive neoplastic lesions that would remain in situ in the rectosigmoid and the number of unnecessary polypectomies that would be avoided (i.e. the number of diminutive non-neoplastic lesions that would be left in situ) is shown. For example, when, in a colonoscopy screening cohort, $90 \%$ of all diminutive adenomas and 
- Table 1 Impact of implementing the leave-in-situ strategy with different proportions of correctly optically diagnosed $\leq 5$-mm adenomas and hyperplastic polyps (HPPs) in a primary colonoscopy screening and fecal immunochemical test (FIT)-positive screening cohort on the overall proportion of correctly diagnosed $\leq 5-\mathrm{mm}$ polyps and the number of (non-)neoplastic lesions left in situ in the rectosigmoid, with histopathology as the gold standard. Results are presented per 1000 individuals with at least one $\leq 5$-mm polyp.

\begin{tabular}{|c|c|c|c|c|c|c|c|}
\hline \multicolumn{2}{|l|}{ Strategy $^{1}$} & \multicolumn{3}{|c|}{ Colonoscopy screening individuals } & \multicolumn{3}{|c|}{ FIT-positive screening individuals } \\
\hline $\begin{array}{l}\text { Correctly } \\
\text { diagnosed } \\
\leq 5-\mathrm{mm} \text { ade- } \\
\text { nomas, \% }\end{array}$ & $\begin{array}{l}\text { Correctly } \\
\text { diagnosed } \\
\leq 5-\mathrm{mm} \\
\text { HPPs, \% }\end{array}$ & $\begin{array}{l}\text { Correctly } \\
\text { diagnosed } \\
\leq 5-\mathrm{mm} \\
\text { polyps, \% }\end{array}$ & $\begin{array}{l}\text { Neoplastic } \\
\text { lesions of } \\
\leq 5 \mathrm{~mm} \text { left in } \\
\text { situ, } \mathrm{n}(\%)^{2}\end{array}$ & $\begin{array}{l}\text { Non-neo- } \\
\text { plastic lesion } \\
\text { of } \leq 5 \mathrm{~mm} \text { left } \\
\text { in situ, } \mathrm{n}(\%)^{3}\end{array}$ & $\begin{array}{l}\text { Correctly } \\
\text { diagnosed } \\
\leq 5-\mathrm{mm} \\
\text { polyps, \% }\end{array}$ & $\begin{array}{l}\text { Neoplastic } \\
\text { lesions of } \\
\leq 5 \mathrm{~mm} \text { left in } \\
\text { situ, } \mathrm{n}(\%)^{2}\end{array}$ & $\begin{array}{l}\text { Non-neo- } \\
\text { plastic lesion } \\
\text { of } \leq 5 \mathrm{~mm} \text { left } \\
\text { in situ, } \mathrm{n}(\%)^{3}\end{array}$ \\
\hline 60 & 60 & 59 & $80(26 \%)$ & $243(39 \%)$ & 60 & $143(26 \%)$ & $137(39 \%)$ \\
\hline 60 & 80 & 68 & $80(26 \%)$ & $324(52 \%)$ & 64 & $143(26 \%)$ & $182(52 \%)$ \\
\hline 60 & 100 & 78 & $80(26 \%)$ & $404(65 \%)$ & 69 & $143(26 \%)$ & $227(65 \%)$ \\
\hline 70 & 60 & 63 & $62(20 \%)$ & $243(39 \%)$ & 67 & $107(20 \%)$ & $137(39 \%)$ \\
\hline 70 & 80 & 73 & $62(20 \%)$ & $324(52 \%)$ & 72 & $107(20 \%)$ & $182(52 \%)$ \\
\hline 70 & 100 & 83 & $62(20 \%)$ & $404(65 \%)$ & 77 & $107(20 \%)$ & $227(65 \%)$ \\
\hline 80 & 60 & 68 & $44(14 \%)$ & $243(39 \%)$ & 74 & $72(14 \%)$ & $137(39 \%)$ \\
\hline 80 & 80 & 78 & 44 (14\%) & $324(52 \%)$ & 79 & $72(14 \%)$ & $182(52 \%)$ \\
\hline 80 & 100 & 87 & 44 (14\%) & $404(65 \%)$ & 84 & $72(14 \%)$ & $227(65 \%)$ \\
\hline 90 & 60 & 73 & $26(8 \%)$ & $243(39 \%)$ & 82 & $37(8 \%)$ & 137 (39\%) \\
\hline 90 & 80 & 82 & $26(8 \%)$ & $324(52 \%)$ & 87 & 37 (8\%) & $182(52 \%)$ \\
\hline 90 & 100 & 92 & $26(8 \%)$ & $404(65 \%)$ & 91 & $37(8 \%)$ & 227 (65\%) \\
\hline 100 & 60 & 77 & $8(2 \%)$ & $243(39 \%)$ & 89 & $2(2 \%)$ & 137 (39\%) \\
\hline 100 & 80 & 87 & $8(2 \%)$ & 324 (52\%) & 94 & $2(2 \%)$ & $182(52 \%)$ \\
\hline 100 & 100 & 96 & $8(2 \%)$ & 404 (65\%) & 99 & $2(2 \%)$ & 227 (65\%) \\
\hline
\end{tabular}

$80 \%$ of all diminutive HPPs were diagnosed correctly by optical diagnosis, 26 diminutive neoplastic lesions would remain in situ in the rectosigmoid per 1000 patients (i. e. one diminutive neoplastic lesion in every 38 patients with a diminutive lesion), representing $8 \%$ of all diminutive neoplastic rectosigmoid lesions. In addition, 324 unnecessary polypectomies would be avoided per 1000 patients, representing a $52 \%$ reduction in unnecessary polypectomies (i.e. diminutive non-neoplastic lesions that would be left in situ).

Table $1 \mathrm{~s}$ (see online-only Supplementary material) shows the impact on the number of diminutive neoplastic lesions that would remain in situ in the rectosigmoid and the number of unnecessary polypectomies that would be avoided if patients without a polyp were also included.

In addition, this simulation study showed that increasing and decreasing the proportion of high confidence diagnoses of all types of diminutive polyps by $10 \%$ barely affected the clinical outcome (i.e. there was hardly any impact on the short-term clinical consequences). As the malignant potential of SSLS is increasingly being emphasized, in this simulation approach, the impact of correctly optically diagnosing diminutive SSLS was also assessed $[21,22]$. The simulation showed that the proportion of correctly diagnosed diminutive SSLs barely affects surveillance interval agreement and the number of (non)neoplastic lesions that would remain in situ. The fact that diminutive SSLs have little relevance is likely to be because of their low prevalence. In addition, individuals often have synchronous adenomas and/or large lesions found at colonoscopy that predominantly determine the surveillance intervals. However, the influence of SSLs on surveillance and the number of SSLs that would remain in situ might expand in the future as the detection rates of SSLS are currently increasing owing to increasing awareness of these subtle lesions among endoscopists [23].

\section{Potential impact on long-term consequences}

The impact of implementing the leave-in-situ strategy on the long-term consequences, such as increase in CRC incidence, CRC mortality, and cost-effectiveness, should be considered. Two studies have evaluated the implementation of the full optical diagnosis strategy (i. e. leave-in-situ and resect-and-discard) $[9,10]$ ( $\triangleright$ Table 2). 
Using the Adenoma and Serrated pathway to Colorectal CAncer (ASCCA) model, Vleugels et al. simulated a biennial FIT screening program, in which they compared an optical diagnosis strategy with the current strategy, submitting all diminutive polyps for histopathology assessment [9]. Implementation of an optical diagnosis strategy in a FIT-based screening program led to one CRC case, one additional CRC death in a cohort of 10000 individuals, and a cost-saving of $€ 6$ per individual.

The National Institute for Health and Care Excellence (NICE) diagnostic guidance on "Virtual chromoendoscopy to assess colorectal polyps during colonoscopy” published in 2017 [10, 24] modelled CRC risk and economic consequences of adopting the complete optical diagnosis strategy using a range of narrowed spectrum technologies (narrow-band imaging [NBI], flexible spectral imaging color enhancement [FICE], iSCAN). Very similar lifetime risks of CRC were reported using histopathology $(3.025 \%)$ versus narrowed spectrum-based optical diagnosis $(3.020 \%-3.045 \%)$ to determine surveillance intervals. NBI and iSCAN were shown to be economically dominant to histopathology in all scenario analyses. Therefore, both modelling studies demonstrate that implementing the optical diagnosis strategy (which includes the leave-in-situ strategy) only marginally influenced long-term outcomes, such as CRC incidence and mortality, whilst saving costs.

\section{Risk of leaving in situ a diminutive lesion with advanced neoplasia}

The risk of cancer in diminutive polyps is very low and estimated to be roughly 1 in 3000 [26]. However, this estimate is not evenly distributed across all populations undergoing colonoscopy. In a pooled review consisting of five FIT cohorts and seven colonoscopy cohorts, a greater prevalence of advanced histology was seen in the FIT cohorts compared with the colonoscopy cohorts across all histological subtypes, although for CRC and villous histology the differences were not statistically significant [4]. In the FIT and colonoscopy cohorts, the pooled prevalence of advanced histology within diminutive polyps was $7.1 \%$ and $1.5 \%(P=0.04)$. The pooled prevalence of CRC was $0.08 \%$ in the FIT cohorts and $0.01 \%$ in the colonoscopy cohorts $(P=$ $0.37)$. This suggests that there may be a difference in the rates of advanced histology in diminutive polyps depending on the indication for colonoscopy.

Both the progression of untreated diminutive adenomas and the incidence of newly developed lesions should be considered when evaluating the incidence of advanced neoplasia (i.e. the risk of leaving in situ a diminutive lesion with advanced neoplasia). Longitudinal follow-up data on individuals with untreated diminutive adenomas are scarce. However, in the Japanese series of Sekiguchi et al. [27], the 5-year cumulative incidence of advanced neoplasia in individuals with untreated diminutive adenomas diagnosed by magnification was $1.4 \%$ (95\% Cl $0.5 \%$ $3.4 \%)$. Of the 508 untreated diminutive adenomas, none of the lesions progressed to advanced neoplasia during the follow-up period, all of the detected advanced neoplastic lesions $(n=21)$ were newly diagnosed in a different location from that in which the untreated diminutive adenoma was originally found.

A systematic review in 2017, including three studies with 327 patients, also showed that untreated diminutive adenomas have an indolent and benign course [7]. Only $0.6 \%$ of all adenomas (2/340) developed into advanced adenomas in 2 to 3 years. Another Japanese study, that of Ninomiya, confirmed these low numbers when using magnification [28]. In their study with 706 patients with diminutive polyps on initial colonoscopy (excluding depressed lesions and Kudo V pit pattern), only two T1 cancers were detected on surveillance colonoscopy, and both were treated radically by endoscopic resection.

Data show that polyps are not equal in terms of their cancer risk. In particular, if there is a depressed area in the polyp, this

- Table 2 Overview of optical diagnosis modelling studies: base-case assumptions, increase colorectal cancer (CRC) burden, and lifetime costsavings when implementing the optical diagnosis strategy calculated per individual.

\begin{tabular}{|c|c|c|c|c|c|c|c|}
\hline \multirow[t]{2}{*}{ Study } & \multirow[t]{2}{*}{$\begin{array}{l}\text { Screening } \\
\text { program }\end{array}$} & \multicolumn{3}{|c|}{$\begin{array}{l}\text { Base-case assumptions for optical diagnosis of } \\
\text { diminutive colorectal polyps }\end{array}$} & \multirow[t]{2}{*}{$\begin{array}{l}\text { Cost-savings } \\
\text { per individual }\end{array}$} & \multirow{2}{*}{$\begin{array}{l}\text { Increased } \\
\text { lifetime risk } \\
\text { of CRC }\end{array}$} & \multirow{2}{*}{$\begin{array}{l}\text { Increased } \\
\text { lifetime } \\
\text { mortality } \\
\text { risk from } \\
\text { CRC }\end{array}$} \\
\hline & & $\begin{array}{l}\text { Sensitivity } \\
\text { for neoplastic } \\
\text { lesions }\end{array}$ & $\begin{array}{l}\text { Sensitivity } \\
\text { for non-neo- } \\
\text { plastic lesions }\end{array}$ & $\begin{array}{l}\text { High con- } \\
\text { fidence pre- } \\
\text { dictions }\end{array}$ & & & \\
\hline $\begin{array}{l}\text { Vleugels et al. } \\
\text { [9] }\end{array}$ & FIT screening & $\begin{array}{l}92 \% \text { adenomas; } \\
91 \% \text { SSLs }\end{array}$ & $88 \%$ & $76 \%$ & $€ 6$ & $0.00466 \%$ & $0.00141 \%$ \\
\hline $\begin{array}{l}\text { NICE (2017) } \\
\text { Picot et al. } \\
{[10,24]}\end{array}$ & FIT screening & $\begin{array}{l}\text { NBI } 91 \% ; \\
\text { FICE } 81.4 \% ; \\
\text { iSCAN } 96.2 \%\end{array}$ & $\begin{array}{l}\text { NBI } 81.9 \% \\
\text { FICE } 85.0 \% \text {; } \\
\text { iSCAN } 90.6 \%\end{array}$ & $78.6 \%$ & $\begin{array}{l}\text { NBI } £ 6.11 ; \\
\text { FICE } £ 887.70 ; \\
\text { iSCAN } £ 8.49\end{array}$ & $\begin{array}{l}\text { NBI } 0.005 \% \\
\text { FICE } 0.02 \% \\
\text { iSCAN } 0.004 \%\end{array}$ & NR \\
\hline $\begin{array}{l}\text { Kessler et al. }{ }^{1} \\
{[25]}\end{array}$ & $\begin{array}{l}\text { Primary colo- } \\
\text { noscopy }\end{array}$ & $90 \%$ & $90 \%$ & $100 \%$ & $\$ 174$ & $0.0076 \%^{2}$ & \\
\hline $\begin{array}{l}\text { Hassan et al. }{ }^{1} \\
{[8]}\end{array}$ & $\begin{array}{l}\text { Primary colo- } \\
\text { noscopy }\end{array}$ & $94 \%$ & $89 \%$ & $83 \%$ & $\$ 25$ & NR & NR \\
\hline
\end{tabular}


substantially increases the risk that there might be a malignant component. Given the negligible risk that diminutive polyps harbor cancer, few data are available on this matter. Recently Oka et al. [29] presented a large study, in which the risk of submucosal invasion of diminutive adenomas was $0.19 \%(15 /$ 7801). Of these 15 lesions, 11 had a depressed area and only $0.01 \%$ of non-depressed diminutive adenomas (1/7687) had deep submucosal invasion. Therefore, if depressed adenomas were excluded from the leave-in-situ strategy, the risk of leaving in situ a cancerous lesion would be greatly reduced. Because of the higher prevalence of advanced histology that has been described, the panel encourages increased detection of non-polypoid and depressed lesions. These lesions should be resected and referred to the pathologist.

\section{Pathological accuracy and reproducibility}

Despite histology being the reference standard for differentiating between polyp subtypes, it is also hampered by some degree of misdiagnosis owing to error in sampling or retrieval, or pathology diagnosis [30-32]. Therefore, a $10 \%$ error rate in the pathological discrimination between polyp subtypes may be assumed unless an enhanced reference standard is used, such as specialist review from multiple pathologists combined with cutting all available tissue. When replacing a pathological with an endoscopic diagnosis, it is preferable that this error rate should not be increased; most studies however have not used an enhanced reference standard.

Furthermore, as a starting point, most studies assume that every polyp is retrieved and available for histological analysis. However, even at expert centers, up to $10 \%$ of polyps may be lost or destroyed after resection. The standard approach to lost or destroyed polyps is to assume that these are adenomas; however, reanalysis from the original DISCARD study suggests that, when taking this issue into account, the lower accuracy of optical diagnosis (versus a non-enhanced pathological reference standard) is balanced by the loss of polyps available for pathological diagnosis that have to be (over)called as adenomas. Therefore, in clinical practice, optical diagnosis and pathology may have similar accuracy on a "per polyp detected" basis [33].

\section{Attitudes of endoscopists and patients with regards to a leave-in-situ strategy}

Willems et al. performed an international survey among 808 endoscopists from nine endoscopy societies to evaluate their attitudes and practices with regards to a leave-in-situ strategy [34] In total, $63 \%$ of the participants partly or completely agreed that diminutive polyps could be left unresected until the next screening colonoscopy because of the low associated cancer risk. Endoscopists were evenly split on the effects of leaving such polyps unresected, with about $50 \%$ thinking that leaving diminutive polyps in place would increase the cancer risk of patients. Moreover, $52 \%$ of endoscopists were already leaving diminutive polyps that appeared non-neoplastic in situ in their daily practice. These results are somewhat different from a survey by Gellad et al. in 2013, which reported that the majority of endoscopists would be somewhat agreeable to leaving diminutive polyps in place if guidelines were to support this practice [35].

Little is known regarding whether patients would accept a leave-in-situ strategy. The only evidence comes from von Renteln et al. who performed a study among 557 patients to investigate the valuable question of whether patients would find a leave-in-situ practice acceptable [36]. They found that approximately $50 \%$ of individuals undergoing a routine colonoscopy would be agreeable to deferring resection of diminutive polyps until the next surveillance colonoscopy and participating in a trial to evaluate this approach.

\section{Resect-and-discard strategy}

\section{RECOMMENDATION}

2 In order to implement the resect-and-discard strategy for colorectal lesions of $1-5 \mathrm{~mm}$, it is clinically acceptable if, during real-time colonoscopy, at least $80 \%$ sensitivity and $80 \%$ specificity is achieved for high confidence endoscopic characterization of colorectal neoplasia of 1$5 \mathrm{~mm}$. Histopathology is used as the gold standard. Level of agreement $100 \%$ (17 Strongly agree; 5 Agree; 0 Neither agree nor disagree; 0 Disagree; 0 Strongly disagree).

The panel took into account a number of considerations in the development of this SODA competence standard for implementation of the resect-and-discard strategy, which are detailed in the following sections.

\section{Potential impact on short-term consequences}

When applying the resect-and-discard part of the optical diagnosis strategy, not recognizing neoplastic diminutive lesions could result in longer than appropriate surveillance intervals, whereas not recognizing non-neoplastic diminutive lesions could result in shorter than appropriate surveillance intervals. Again, it could be argued that the correct diagnosis of each polyp subtype is not of equal clinical importance. The clinical implications of incorrectly diagnosing neoplastic lesions (i.e. a longer surveillance interval) and incorrectly diagnosing nonneoplastic lesions (i. e. a shorter surveillance interval) are completely different. Therefore, competence standards should take both scenarios into account.

The aforementioned simulation study also determined the relationship between the proportion of correctly optically diagnosed diminutive polyps (adenomas and HPPs) and the surveillance interval agreement when implementing the resect-anddiscard strategy in a primary colonoscopy and FIT-positive screening cohort ( $>$ Table 3 ; Tables $2 \mathbf{s}$ and $\mathbf{3 s}$ ). Results in the tables are presented per 1000 individuals with at least one diminutive polyp. The impact on surveillance interval agreement is indicated by showing the proportion in agreement with the US Multi-Society Task Force on Colorectal Cancer surveillance guideline [37] and the proportion in agreement with the ESGE surveillance guideline [38]. For example, if $80 \%$ of all diminu- 
tive adenomas and $80 \%$ of all diminutive HPPs were to be diagnosed correctly with optical diagnosis in the primary colonoscopy screening program (i.e. $78 \%$ of all diminutive polyps correctly diagnosed), the proposed surveillance intervals would agree with those determined by histological analysis for $80 \%$ of the cases when using the US guideline and for $95 \%$ of the cases when using the ESGE guideline.

- Fig. 1 shows the impact of implementing the resect-anddiscard strategy for colorectal lesions of $1-5 \mathrm{~mm}$ with $80 \%$ sensitivity and $80 \%$ specificity for high confidence endoscopic characterization of $1-5 \mathrm{~mm}$ colorectal neoplasia on surveillance interval agreements. Different thresholds for the proportion of correctly diagnosed diminutive polyps again lead to different proportions in agreement with the surveillance guidelines depending on the guidelines and the clinical setting.

\section{Potential impact on long-term consequences}

Data on the long-term clinical consequences and cost-effectiveness of implementing the resect-and-discard strategy are limited to four modelling studies ( $\vee$ Table 2 ). Two of these evaluated the implementation of the complete optical diagnosis strategy and demonstrated that implementation only marginally influenced long-term outcomes, such as CRC incidence and mortality, whilst saving costs $[1,9,33]$. Two studies exclusively evaluated the implementation of the resect-and-discard strategy $[8,25]$. In the modelling study by Kessler et al. [25], the estimated cost-savings of implementing the resect-and-discard strategy in a primary colonoscopy setting was $\$ 174$ per individual, with a number needed to harm because of missed interval cancer of 7979. In the modelling study by Hassan et al., adoption of the resect-and-discard strategy in a primary colonoscopy screening program resulted in a saving of $\$ 25$ per individual, without any meaningful effect on screening efficacy [8]. In summary, the modelling studies described above have provided evidence that implementation of the optical diagnosis strategy is associated with substantial cost-savings with negligible impact on patients' cancer risk.

Data on the impact of varying the rate of high confidence diagnosis on long-term clinical consequences are limited to two modelling studies. These studies showed that varying the proportion of high confidence diagnoses did not increase the CRC burden $[8,9]$. They demonstrate that the cost-effective-

- Table 3 Impact of implementing the resect and-discard strategy with different proportions of correctly optically diagnosed $\leq 5$-mm adenomas and hyperplastic polyps (HPPs) in a primary colonoscopy screening and fecal immunochemical test (FIT)-positive cohort on the overall proportion of correctly diagnosed $\leq 5$-mm polyps and surveillance interval agreement with histopathology as the gold standard.

Results are presented per 1000 individuals with at least one $\leq 5$-mm polyp.

\begin{tabular}{|c|c|c|c|c|c|c|c|}
\hline \multicolumn{2}{|l|}{ Strategy* } & \multicolumn{3}{|c|}{ Colonoscopy screening individuals } & \multicolumn{3}{|c|}{ FIT-positive screening individuals } \\
\hline \multirow{2}{*}{$\begin{array}{l}\text { Correctly diag- } \\
\text { nosed } \leq 5-\mathrm{mm} \\
\text { adenomas, } \%\end{array}$} & \multirow{2}{*}{$\begin{array}{l}\text { Correctly diag- } \\
\text { nosed } \leq 5-\mathrm{mm} \\
\text { HPPs, \% }\end{array}$} & \multirow{2}{*}{$\begin{array}{l}\text { Correctly diag- } \\
\text { nosed } \leq 5-\mathrm{mm} \\
\text { polyps, } \%\end{array}$} & \multicolumn{2}{|c|}{$\begin{array}{l}\text { Surveillance interval } \\
\text { agreement, \% }\end{array}$} & \multirow{2}{*}{$\begin{array}{l}\text { Correctly diag- } \\
\text { nosed } \leq 5-\mathrm{mm} \\
\text { polyps, } \%\end{array}$} & \multicolumn{2}{|c|}{$\begin{array}{l}\text { Surveillance interval } \\
\text { agreement, \% }\end{array}$} \\
\hline & & & ESGE & US & & ESGE & US \\
\hline 60 & 60 & 60 & 95 & 66 & 60 & 97 & 84 \\
\hline 60 & 80 & 68 & 95 & 73 & 64 & 97 & 86 \\
\hline 60 & 100 & 78 & 95 & 80 & 69 & 97 & 88 \\
\hline 70 & 60 & 63 & 95 & 69 & 67 & 98 & 87 \\
\hline 70 & 80 & 73 & 95 & 76 & 72 & 98 & 89 \\
\hline 70 & 100 & 83 & 95 & 84 & 77 & 98 & 91 \\
\hline 80 & 60 & 68 & 95 & 73 & 74 & 98 & 90 \\
\hline 80 & 80 & 78 & 95 & 80 & 79 & 98 & 91 \\
\hline 80 & 100 & 87 & 95 & 88 & 84 & 98 & 93 \\
\hline 90 & 60 & 73 & 95 & 76 & 82 & 99 & 92 \\
\hline 90 & 80 & 82 & 96 & 83 & 87 & 99 & 94 \\
\hline 90 & 100 & 92 & 96 & 92 & 91 & 99 & 96 \\
\hline 100 & 60 & 77 & 96 & 79 & 89 & 99 & 94 \\
\hline 100 & 80 & 87 & 96 & 87 & 94 & 100 & 97 \\
\hline 100 & 100 & 96 & 96 & 96 & 99 & 100 & 99 \\
\hline \multicolumn{8}{|c|}{$\begin{array}{l}\text { ESGE, European Society of Gastrointestinal Endoscopy (ESGE) post-polypectomy surveillance guidelines; US, US Multi-Society Task Force on Colorectal Cancer } \\
\text { guidelines. }\end{array}$} \\
\hline
\end{tabular}




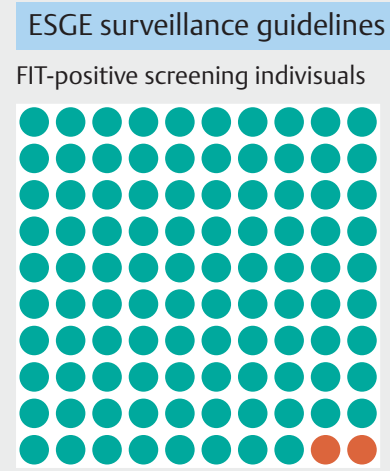

Primary colonoscopy screening individuals

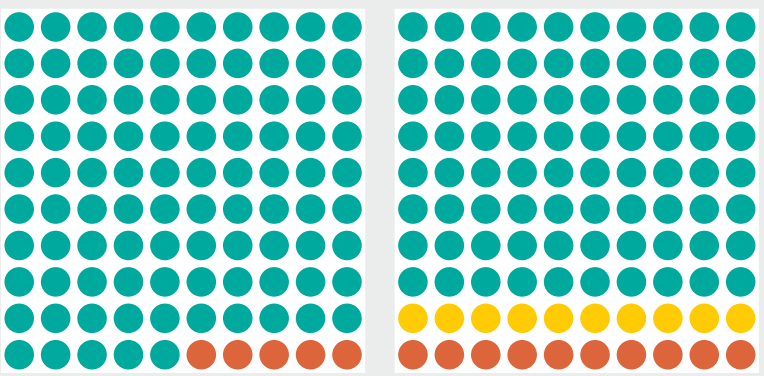

Agreemant in surveillance interval assignment

Shorter surveillance interval assignment

Longer surveillance interval assignment

- Fig. 1 Impact of implementing the resect-and-discard strategy for colorectal lesions of $1-5 \mathrm{~mm}$ with $80 \%$ sensitivity and $80 \%$ specificity for high confidence endoscopic characterization of $1-5 \mathrm{~mm}$ colorectal neoplasia on surveillance interval agreement using the ESGE surveillance guideline [38] and US Multi-Society Task Force on Colorectal Cancer surveillance guideline [37].

Histopathology is used as the gold standard. Results are presented per 100 individuals.

ness of the optical diagnosis strategy appears to be dependent on the proportion of high confidence diagnoses [39]. Hassan et al. showed a linear relationship between the rate of high confidence prediction and the undiscounted savings projected in the US population, when implementing the optical diagnosis strategy in a colonoscopy screening program [8]. Assuming a $100 \%$ (best-case scenario) and 50\% (worst-case scenario) proportion of high confidence diagnoses, the undiscounted benefit for the US population would be $\$ 40$ million and $\$ 20$ million, respectively. In another simulation study, varying the proportion of high confidence diagnoses from $50 \%$ to $100 \%$ led to cost-savings of $€ 5$ and $€ 7$, respectively, per individual in a biennial FIT screening program [9].

\section{Risk of metastatic disease after resecting and discarding} a diminutive lesion with cancer

The risk of metastatic disease after resecting and discarding a diminutive polyp in situ that contained cancer is very limited. In the study of Oka et al. [29], for seven diminutive invasive polyp cancers that were treated surgically, no lymph node metastases were seen.

Attitudes of endoscopists and patients with regards to a resect-and-discard strategy

When Willems et al. asked endoscopists in their worldwide survey about barriers to implementation of a resect-and-discard strategy, it appears the clinical uptake of resect-and-discard is low [34]. They found that only $15.8 \%$ of the endoscopists used the resect-and-discard strategy in their current practice and $59.9 \%$ thought that implementation of the resect-and-discard strategy was not feasible in its current form. Of all endoscopists, $44.6 \%$ were afraid of making a wrong diagnosis, 53.8 $\%$ were concerned about potential medicolegal issues, and $58.3 \%$ were afraid of assigning incorrect surveillance intervals to patients. These findings are similar to those of Soudagar et al. in 2016, where medicolegal concerns were the main barrier to implementation of the resect-and-discard strategy for the 105 gastroenterologists surveyed during a national conference in the USA [40]. While the consensus for most regions was that resect-and-discard was not feasible, $54 \%$ of the European endoscopists showed an increased adoption of the strategy.

Rex et al. surveyed American colonoscopy patients and found that $66 \%$ of them would accept a resect-and-discard strategy [26]. Of those unwilling to accept resect-and-discard, $50 \%$ wanted an absolute zero chance of cancer in diminutive polyps and were willing to pay out of their own pocket for histological assessment of these diminutive polyps. Vu et al. further assessed this by approaching patients with the hypothetical question of whether they would be willing to pay approximately $\$ 150$ for pathology rather than use the resect-and-discard strategy if the risk of a cancer in their polyp was $1: 3000$ [41]. Over two-thirds of patients would be willing to pay to have their diminutive polyp sent for pathological evaluation.

\section{Conclusion and future prospects}

This ESGE Position Statement provides new competence standards for the optical diagnosis of diminutive colorectal polyps that are clinically acceptable, achievable, and easy to measure in daily practice (SODA standards). $>$ Fig. 2 provides a flowchart of the application of the optical diagnosis strategy using the SODA competency standards for diminutive colorectal polyps. The development of these standards is based on the currently available evidence and a Delphi-based consensus process undertaken by a designated ESCE task force. These new standards facilitate implementation of the optical diagnosis strategy in daily practice. However, an accreditation and monitoring scheme should be set up to assess competence and audit performance. In addition, these new clinically based standards clearly define diagnostic performance thresholds for optical diagnosis of diminutive polyps by artificial intelligence systems in relation to the clinical actions and consequences. 


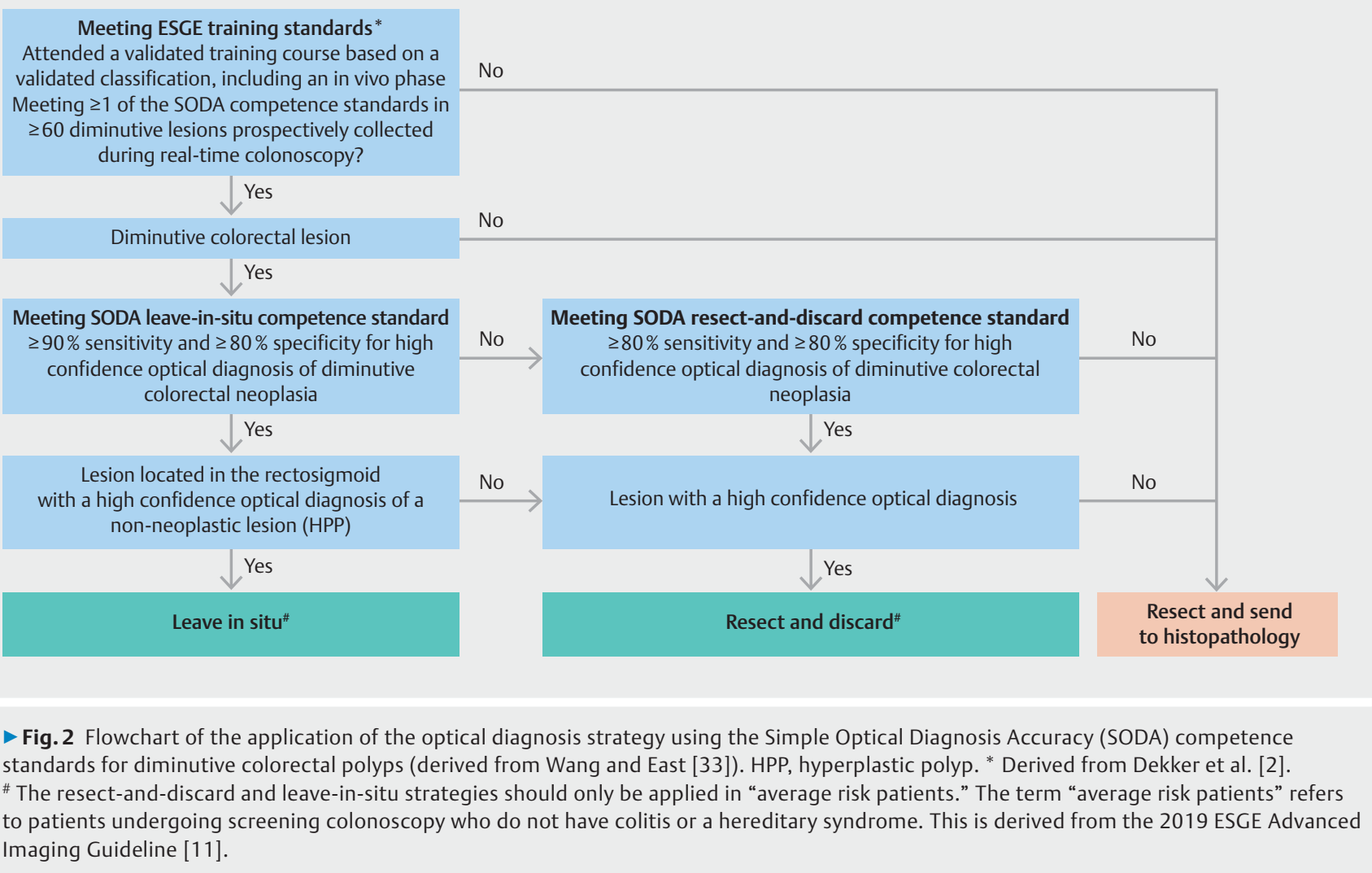

\section{Disclaimer}

ESGE Guidelines and Position Statements represent a consensus of best practice based on the available evidence at the time of preparation. They might not apply in all situations and should be interpreted in the light of specific clinical situations and resource availability. Further controlled clinical studies may be needed to clarify aspects of these statements, and revision may be necessary as new data appear. Clinical considerations may justify a course of action at variance with these recommendations. ESGE Guidelines and Position Statements are intended to be an educational device providing information that may assist endoscopists in providing care to patients. They are not rules and should not be construed as establishing a legal standard of care or as encouraging, advocating, requiring, or discouraging any particular treatment.

\section{Acknowledgments}

R. Bisschops is supported by the Research Foundation - Flanders (FWO). J.E. East is funded by the National Institute for Health Research (NIHR) Oxford Biomedical Research Centre. The views expressed are those of the authors and not necessarily those of the National Health Service, the NIHR, or the Department of Health. 
Competing interests

R. Bisschops has received research support from Cook and Medtronic, and financial support for symposium organization from Cook, Boston Scientific, Olympus, and Erbe (2009-2109), and speakers' fees from Boston Scientific and Medtronic (2009-2019). E. Coron has received speaker's fees from Fujifilm (2018-2020). E. Dekker has received speaker's fees from Roche (2018), Norgine (2019), Olympus and GI Supply (both 2019 to 2020), and Fujifilm (2020), and has provided consultancy to Fujifilm (2018), CPP-FAP (2019), GI Supply (2019 to 2020), Olympus (2020 to present), PAION and Ambu (both 2021); she received a research grant from Fujifilm (2017 to 2020) and her department has equipment on loan from Fujifilm (2017 to present) and Olympus (2021). M. Dinis-Ribeiro receives an educational grant from Olympus (2020 to present) and a research grant from Fujifilm (2020 to present); he is co-editor in-chief of Endoscopy. J.E. East has provided consultancy to, and holds share options in, Satisfai Health (2020 to present). C. Hassan has received research support from Fujifilm (2017 to present); his department has received support from Sonoscape. M. lacucci receives research support from Pentax (2011 to present), Olympus (2017 to present) and Fujifilm (2018 to present). Y. Mori receives consultancy and speaker's fees from Olympus (2018 to present) and has an ownership interest in Cybernet System Corp. (2020 to present). H. Neumann has provided consultancy to Fujifilm, Sonoscope, and Boston Scientific (all 2019 to 2020). M. Pellisé has received consultancy and speaker's fees from Norgine Iberia (2015 to 2020), a consultancy fee from GI Supply (2019), speaker's fees from Casen Recordati (2016 to 2019), Olympus (2018), and Jansen (2018), and research funding from Fujifilm Spain (2019), Fujifilm Europe (2020), and Casen Recordati (2020); her department has received loan material from Fujifilm Spain (2017 to present), a research grant from Olympus Europe (2005 to 2019), and loan material and a research grant from Fujifilm Europe (2020 to 2021); she is a Board member of ESGE and SEED, and is a co-editor of Endoscopy (2015 to 2021). I. Puig has provided advisory services to Fujifilm (2020 to present) and has equipment on loan from Olympus and Fujifilm (both 2019 to present). B. Saunders receives research funding from Olympus (2019 to present). D.J. Tate received an educational grant from Olympus (2018 to 2019). G. Antonelli, M. Bustamante-Balén, G. Cortas, V.M.H. Coupé, D.E. Dobru, M.J.E. Greuter, Y. Hazewinkel, B.B.S.L. Houwen, R. Jover, R. Kuvaev, G. Longcroft-Wheaton, M.D. Rutter, and J.L.A. Vleugels declare that they have no conflict of interest.

\section{References}

[1] Bisschops R, Dekker E, East JE et al. European Society of Gastrointestinal Endoscopy (ESGE) curricula development for postgraduate training in advanced endoscopic procedures: rationale and methodology. Endoscopy 2019; 51: 976-979

[2] Dekker E, Houwen B, Puig I et al. Curriculum for optical diagnosis training in Europe: European Society of Gastrointestinal Endoscopy (ESGE) Position Statement. Endoscopy 2020; 52: 899-923

[3] Pickhardt PJ, Kim DH. Colorectal cancer screening with CT colonography: key concepts regarding polyp prevalence, size, histology, morphology, and natural history. AJR Am J Roentgenol 2009; 193: 40-46

[4] Vleugels JLA, Hassan C, Senore C et al. Diminutive polyps with advanced histologic features do not increase risk for metachronous advanced colon neoplasia. Gastroenterology 2019; 156: 623634.e623

[5] Ignjatovic A, East JE, Suzuki N et al. Optical diagnosis of small colorectal polyps at routine colonoscopy (Detect InSpect ChAracterise Resect and Discard; DISCARD trial): a prospective cohort study. Lancet Oncol 2009; 10: 1171-1178
[6] Hassan C, Pickhardt PJ, Kim DH et al. Systematic review: distribution of advanced neoplasia according to polyp size at screening colonoscopy. Aliment Pharmacol Ther 2010; 31: 210-217

[7] Vleugels JLA, Hazewinkel Y, Fockens P et al. Natural history of diminutive and small colorectal polyps: a systematic literature review. Gastrointest Endosc 2017; 85: 1169-1176.e1161

[8] Hassan C, Pickhardt PJ, Rex DK. A resect and discard strategy would improve cost-effectiveness of colorectal cancer screening. Clin Gastroenterol Hepatol 2010; 8: 865-869.e861-e863

[9] Vleugels JLA, Greuter MJE, Hazewinkel Y et al. Implementation of an optical diagnosis strategy saves costs and does not impair clinical outcomes of a fecal immunochemical test-based colorectal cancer screening program. Endosc Int Open 2017; 5: E1197-E1207

[10] National Institute for Health and Care Excellence. Virtual chromoendoscopy to assess colorectal polyps during colonoscopy. Diagnostics guidance [DG28]. 2017: Available from (Accessed 13.10.2021): https://www.nice.org.uk/guidance/dg28

[11] Bisschops R, East JE, Hassan C et al. Advanced imaging for detection and differentiation of colorectal neoplasia: European Society of Gastrointestinal Endoscopy (ESGE) Guideline - Update 2019. Endoscopy 2019; 51: 1155-1179

[12] Rutter MD, East J, Rees C] et al. British Society of Gastroenterology/ Association of Coloproctology of Great Britain and Ireland/Public Health England post-polypectomy and post-colorectal cancer resection surveillance guidelines. Gut 2020; 69: 201-223

[13] Jones ], Hunter D. Consensus methods for medical and health services research. BMJ 1995; 311: 376

[14] Boulkedid R, Abdoul H, Loustau M et al. Using and reporting the Delphi method for selecting healthcare quality indicators: a systematic review. PloS One 2011; 6: e20476

[15] Humphrey-Murto $S$, de Wit M. The Delphi method-more research please. J Clin Epidemiol 2019; 106: 136-139

[16] Houwen BBSL, Greuter MJ, Vleugels JLA et al. Guidance for setting easy-to-adopt competence criteria for optical diagnosis of diminutive colorectal polyps: a simulation approach. Gastrointest Endosc 2021; 94: 812-822.e4

[17] Houwen BBSL, Hassan C, Hazewinkel Y et al. Methodological framework for the development of standards for optical diagnosis in gastrointestinal endoscopy: European Society of Gastrointestinal Endoscopy (ESGE) Position Statement. Endoscopy 2021: doi:10.1055/a1689-5615

[18] Stoop EM, de Haan MC, de Wijkerslooth TR et al. Participation and yield of colonoscopy versus non-cathartic CT colonography in population-based screening for colorectal cancer: a randomised controlled trial. Lancet Oncol 2012; 13: 55-64

[19] Vleugels JLA, Dijkgraaf MGW, Hazewinkel Y et al. Effects of training and feedback on accuracy of predicting rectosigmoid neoplastic lesions and selection of surveillance intervals by endoscopists performing optical diagnosis of diminutive polyps. Gastroenterology 2018; 154: 1682-1693.e1681

[20] Toes-Zoutendijk E, van Leerdam ME, Dekker E et al. Real-time monitoring of results during first year of Dutch Colorectal Cancer Screening Program and optimization by altering fecal immunochemical test cut-off levels. Gastroenterology 2017; 152: 767-775.e762

[21] Jass JR. Classification of colorectal cancer based on correlation of clinical, morphological and molecular features. Histopathology 2007; 50: 113-130

[22] Toyota M, Ahuja N, Ohe-Toyota M et al. CpG island methylator phenotype in colorectal cancer. Proc Natl Acad Sci USA 1999; 96: 86818686

[23] Bleijenberg AGC, van Leerdam ME, Bargeman M et al. Substantial and sustained improvement of serrated polyp detection after a simple educational intervention: results from a prospective controlled trial. Gut 2020; 69: 2150-2158 
[24] Picot ], Rose M, Cooper K et al. Virtual chromoendoscopy for the realtime assessment of colorectal polyps in vivo: a systematic review and economic evaluation. Health Technol Assess 2017; 21: 1-308

[25] Kessler WR, Imperiale TF, Klein RW et al. A quantitative assessment of the risks and cost savings of forgoing histologic examination of diminutive polyps. Endoscopy 2011; 43: 683-691

[26] Rex DK, Patel NJ, Vemulapalli KC. A survey of patient acceptance of resect and discard for diminutive polyps. Gastrointest Endosc 2015; 82: 376-380.e371

[27] Sekiguchi M, Otake Y, Kakugawa Y et al. Incidence of advanced colorectal neoplasia in individuals with untreated diminutive colorectal adenomas diagnosed by magnifying image-enhanced endoscopy. Am J Gastroenterol 2019; 114: 964-973

[28] Ninomiya Y, Oka S, Tanaka S et al. Clinical impact of surveillance colonoscopy using magnification without diminutive polyp removal. Dig Endosc 2017; 29: 773-781

[29] Oka S, Tanaka S, Nakadoi K et al. Endoscopic features and management of diminutive colorectal submucosal invasive carcinoma. Dig Endosc 2014; 26: (Suppl. 02): 78-83

[30] Payne SR, Church TR, Wandell M et al. Endoscopic detection of proximal serrated lesions and pathologic identification of sessile serrated adenomas/polyps vary on the basis of center. Clin Gastroenterol Hepatol 2014; 12: 1119-1126

[31] Schachschal G, Sehner S, Choschzick M et al. Impact of reassessment of colonic hyperplastic polyps by expert GI pathologists. Int J Colorectal Dis 2016; 31: 675-683

[32] Mahajan D, Downs-Kelly E, Liu X et al. Reproducibility of the villous component and high-grade dysplasia in colorectal adenomas $<1 \mathrm{~cm}$ : implications for endoscopic surveillance. Am J Surg Pathol 2013; 37 : 427-433
[33] Wang LM, East JE. Diminutive polyp cancers and the DISCARD strategy: Much ado about nothing or the end of the affair? Gastrointest Endosc 2015; 82: 385-388

[34] Willems P, Djinbachian R, Ditisheim S et al. Uptake and barriers for implementation of the resect and discard strategy: an international survey. Endosc Int Open 2020; 8: E684-E692

[35] Gellad ZF, Voils Cl, Lin L et al. Clinical practice variation in the management of diminutive colorectal polyps: results of a national survey of gastroenterologists. Am J Gastroenterol 2013; 108: 873-878

[36] von Renteln D, Bouin M, Barkun AN et al. Patients' willingness to defer resection of diminutive polyps: results of a multicenter survey. Endoscopy 2018; 50: 221-229

[37] Gupta S, Lieberman D, Anderson JC et al. Recommendations for follow-up after colonoscopy and polypectomy: a consensus update by the US Multi-Society Task Force on Colorectal Cancer. Gastroenterology 2020; 158: 1131-1153.e5

[38] Hassan C, Antonelli G, Dumonceau JM et al. Post-polypectomy colonoscopy surveillance: European Society of Gastrointestinal Endoscopy (ESGE) Guideline - Update 2020. Endoscopy 2020; 52: 687-700

[39] Kaltenbach T, Rastogi A, Rouse RV et al. Real-time optical diagnosis for diminutive colorectal polyps using narrow-band imaging: the VALID randomised clinical trial. Gut 2015; 64: 1569-1577

[40] Soudagar AS, Nguyen M, Bhatia A et al. Are gastroenterologists willing to implement the "predict, resect, and discard" management strategy for diminutive colorectal polyps? Results from a national survey J Clin Gastroenterol 2016; 50: e45-e49

[41] Vu HT, Sayuk GS, Gupta N et al. Patient preferences of a resect and discard paradigm. Gastrointest Endosc 2015; 82: 381-384.e381 